\title{
Size effect on thermodynamic properties of free nanocrystals
}

\author{
A. I. Karasevskii* and V. V. Lubashenko ${ }^{\dagger}$ \\ G. V. Kurdyumov Institute for Metal Physics \\ 36 Vernadsky str., Kiev, 03142, Ukraine
}

\begin{abstract}
We demonstrate that the discrete character of the vibrational spectrum of a small crystal accounts for size dependence of its thermodynamic properties and melting temperature. Using a self-consistent statistical method [Phys. Rev. B 66, 054302 (2002)] we derive the Gibbs free energy of free nanocrystalline plates and calculate the thermodynamic parameters as functions of plate thickness for $\mathrm{Cu}$.
\end{abstract}

PACS numbers: 64.70.D-, 65.80.+n

\section{Introduction}

The effect of depression of melting point was first predicted for free nanoparticles by Pawlow [1] from an analysis of their thermodynamic properties. Further, it was proved experimentally in transmission electron microscopy studies of granular films of $\mathrm{Pb}, \mathrm{Sn}$, and $\mathrm{Bi}$ 2. Later, a relationship between the melting temperature of small metallic species and their size was established on the basis of results of electron microscopy studies of $\mathrm{Sn}[3], \mathrm{Pb}$ and In [4, $\mathrm{Ag}, \mathrm{Cu}, \mathrm{Al}$ and $\mathrm{Ge}[5]$ thin films. A comprehensive analysis of electron diffraction data on melting of $\mathrm{Au}$ nanoparticles and their comparison with predictions of phenomenological approaches were carried out in Ref. [6].

${ }^{*}$ E-mail address: akaras@imp.kiev.ua

${ }^{\dagger}$ E-mail address: vilu@imp.kiev.ua 
The electron diffraction methods, providing only the visual picture of melting of nanocrystals, allow one to relate the melting temperature to the particle size, but they give no information about energetic properties of a nanosystem. In order to get more insight into nature of melting of nanocrystals, these methods are often combined with various nanocalorimetric techniques, thus providing a possibility of estimation of both the melting temperature and the latent heat of fusion of nanocrystals [7]-[16].

Early theoretical studies of thermodynamics of nanocrystals were focused on attempts to explain the strong size dependence of the melting temperature. In thermodynamic consideration of an equilibrium between solid and liquid phases, an important role was ascribed to the surface of the nanoparticle [1, 4, 6, 25, 26]. Some phenomenological models of melting of free nanoparticles employed the effect of surface melting inherent in the most of solids. For example, the liquid-layer model [3, 27] postulates formation of a quasi-liquid layer at the surface of a nanocrystal at temperature below its melting point. Among recent theoretical approaches to description of sizedependent melting of nanocrystals, we would like to mention the liquid-drop model [28] taking into account reduction of the average cohesion energy of atoms of a nanoparticle with reduction of its size and using an earlier proposed relation between the melting temperature and the cohesion energy [29].

Recently it was reported that thermodynamic properties of nanocrystals, such as cohesion energy [17, 18], Debye temperature [17, 19, 20], activation energy of diffusion [21, 22, vacancy formation energy [23, 24] etc. display also size dependence. These experimental facts point to size influence on statistical characteristics of atoms.

A considerable advance in computational capacity made for the last decade has enabled first-principles simulation of dynamical behavior and energetic characteristics of systems consisting of more than $10^{5}$ atoms [30]-[38], i.e. nanocrystals in the so-called mesoscale regime.

A comprehensive molecular dynamics studies of thermodynamics of nanosized systems was carried out in Ref. [35] for spherical Cu nanocrystals. The obtained results demonstrate that both melting temperature and the latent heat of fusion decrease smoothly as particle radius decreases. The analysis of energetic and structural properties of nanocrystals revealed existence of a rather thin surface layer where average potential energy and root-meansquare (rms) displacements of atoms are largely different from that of the core region. The rms displacements of atoms in the surface layer increase 
dramatically at temperature below the bulk melting point of the nanoparticle $T_{m}$, indicating melting of this layer. The thickness of the surface layer is relatively small and almost independent of temperature. The rms displacements of bulklike atoms of the nanocrystal rise linearly with temperature, and melting of the inner region of the particle occurs when the Lindemann criterion is fulfilled. Therefore, the molecular dynamics analysis of thermodynamic properties of nanocrystals [35, 36] revealed that a nanosized particle should be regarded as a structurally and energetically heterogeneous system.

According to results obtained in Ref. [35], the behavior of the average potential energy of atoms of a nanocrystal exhibits essentially nonlinear rise near $T_{m}$. As shown in Refs. [39, 40] for the bulk solids, such a behavior is attributed to evolution of anharmonic instability of the phonon system of the crystal which is directly related to the melting transition.

In this work we study size-dependent modification of the phonon spectrum of crystals and its influence on thermodynamic properties. For the sake of simplicity, we restrict ourselves with the case of a nanocrystalline plate. We show that the vibrational spectrum of such a system is substantially discrete and size-dependent. The phonon spectrum determines the parameters of statistical distribution of atomic displacements of the crystal. Thus, the average values of energetic properties of the crystal exhibit size dependence. In our opinion, this is an important mechanism responsible for depression of the melting point and variation of thermodynamic properties of free nanocrystals with decreasing of their size. It is necessary to note that influence of discreteness of the spectrum of eigenvalues on energetic characteristics of many-particle finite systems was considered previously for atomic nuclei [41] and nanoparticles of degenerated semiconductors [42. In these studies it was demonstrated that corrections due to the discreteness of the spectrum are proportional to the inverse size.

\section{Free energy of a crystal}

To examine size influence on thermodynamics of nanocrystals, we use a self-consistent statistical method [43] developed to compute thermodynamic properties of simple solids. The method consists, first, in derivation of a binary distribution function of atomic coordinates and, second, in a variational procedure of computation of interatomic distance and effective parameters of quasi-elastic bonds between atoms of the crystal. It was shown that the 
phonon spectrum of the crystal determines parameters of the distribution function and, therefore, the average values of energetic characteristics of the crystal. Therefore, this approach allows one to clarify how size-dependent modification of the phonon spectrum of a small crystal affects its thermodynamic properties. The method has been applied to description of thermal characteristics of the rare gas crystals (RGC) [43]- 45] and some simple fcc metals [46]. In the framework of this method we also computed the formation energy of a vacancy in the RGC as a function of temperature and demonstrated that approaching the melting point (which is assumed to be directly related to the point of anharmonic instability) is accompanied by dramatic reduction of the energy required to create structure defects of the lattice [39, 40, 47]. As in our previous studies, we assume the interatomic interaction to be pairwise and approximated by the Morse potential

$$
u(r)=A\left[e^{-2 \alpha\left(r-R_{0}\right)}-2 e^{-\alpha\left(r-R_{0}\right)}\right] .
$$

The parameters $A, R_{0}$, and $\alpha$ have been determined so that calculated values of interatomic distance, bulk modulus, and sublimation energy at zero temperature fitted the corresponding experimental data. These parameters are given in Ref. [39] for the RGC and in Ref. [46] for $\mathrm{Cu}$ and $\mathrm{Ag}$.

In this work we restrict ourselves with the high-temperature limit when the distribution function of atomic displacements in a simple crystal may be represented as a product of one-particle Gaussian functions [39] given by

$$
f\left(\mathbf{q}_{i}\right)=C \exp \left(-\frac{\alpha^{2} c^{2} q_{i}^{2} n(\tau)}{\tau}\right),
$$

where $\mathbf{q}_{i}$ is the displacement of an atom from the $i$ th lattice site, $\tau=k_{B} T / A$ is the reduced temperature, $c$ is a dimensionless effective parameter of quasielastic bond of neighboring atoms, and the coefficient $n(\tau)$ determines contribution of the phonon spectrum to the distribution width. At high temperature it is expanded into a power series as

$$
n(\tau)=\sum_{l=0}^{\infty}(-1)^{l} n_{l}\left(\frac{c \Lambda}{\tau}\right)^{2 l}
$$

where

$$
\Lambda=\frac{\hbar \alpha}{\sqrt{M A}}
$$


is the de Boer parameter for the Morse potential, $M$ is atomic mass. The condition for the applicability of the high-temperature approximation is given by inequality $\tau>c \Lambda$. Coefficients $n_{l}$ are determined by the phonon spectrum of the crystal [43]. For example,

$$
\begin{aligned}
& n_{0}=\frac{1}{2} \sum_{j} \int \tilde{\omega}_{j}^{2}(\mathbf{K}) e_{j x}^{2}(\mathbf{K}) d \mathbf{K}, \\
& n_{1}=\frac{1}{24} \sum_{j} \int \tilde{\omega}_{j}^{4}(\mathbf{K}) e_{j x}^{2} d \mathbf{K},
\end{aligned}
$$

where $\mathbf{K}$ is reduced wave vector [43],

$$
\widetilde{\omega}_{j}(\mathbf{k})=\left(\frac{M}{A \alpha^{2} c^{2}}\right)^{1 / 2} \omega_{j}(\mathbf{k})
$$

is the reduced frequency of a phonon with wave vector $\mathbf{k}$ and branch $j$, and $e_{j x}(\mathbf{k})$ is the projection of a phonon polarization vector on the $x$ axis. The integration in Eqs. (5) and (6) is carried out over the first Brillouin zone. For a bulk fcc crystal, $n_{0}=2, n_{1}=5 / 6$. Eqs. (5) -(6) were derived in Ref. [43] for the bulk crystals, but, according to Ref. [48], such an expression is applicable to any finite system of bound harmonic oscillators.

In the high-temperature approximation the Gibbs free energy $\Phi$ of a simple fcc crystal consisting of $N$ atoms can be written as [43, 46, 39]

$$
\begin{aligned}
g(\tau, p, c, b) & =\frac{\Phi}{A N}=\left(\frac{\tau}{3}+3 \tau \log \frac{c \Lambda}{\tau}\right)+\frac{z}{2}\left[e^{-2 b+\frac{2 \tau}{n(\tau) c^{2}}}-2 e^{-b+\frac{\tau}{2 n(\tau) c^{2}}}\right] \\
& -\frac{a_{3} \tau^{2}}{c^{6}}\left[e^{-2 b+\frac{2 \tau}{n(\tau) c^{2}}}-\frac{1}{4} e^{-b+\frac{\tau}{2 n(\tau) c^{2}}}\right]^{2}-\varkappa_{l} \frac{\epsilon}{A}\left(\frac{\sigma}{R}\right)^{6}+p \alpha^{3} v
\end{aligned}
$$

Here $R$ is the nearest neighbor distance, $b=\alpha\left(R-R_{0}\right)$ is a reduced lattice expansion, $z=12$ is the coordination number, $p=P / \alpha^{3} A$ is reduced external pressure, and $v=R^{3} / \sqrt{2}$ is volume per atom.

The first term in (8) determines the entropy part of the free energy of atomic vibrations in the crystal, the second term represents the average potential energy of interaction of neighboring atoms. The third term determines a contribution to the free energy of the crystal due to the cubic anharmonicity of collective atomic vibrations, evaluated in the second order of the perturbation theory $\left(a_{3} \approx 2.31\right.$ for the RGC [39, 40], $a_{3} \approx 2.9$ for $\mathrm{Cu}$, and $a_{3} \approx 2.0$ 
for $\mathrm{Ag}$ [46]). The next term takes into account the long-range attraction between atoms of a molecular crystal, where $\varepsilon$ and $\sigma$ are the parameters of the Lennard-Jones potential and $\varkappa_{l}=4.91$ for the fcc lattice [39]. In the case of simple metals, this term should be replaced with an electron gas contribution to the kinetic energy of the crystal [46],

$$
\varepsilon_{\mathrm{el}}=\frac{3}{5}\left(\frac{3 \pi^{2}}{2}\right)^{2 / 3} \frac{\hbar^{2}}{m R^{2} A},
$$

where $m$ is the effective electron mass.

\section{Vibrational spectrum of a thin plate}

To determine size contribution to the free energy of a nanocrystal of size $h$ $(h \gg R$ ), we proceed from the assumption that the size-dependent modification of the vibrational spectrum is most pronounced in its long-wave part, with the wave vectors $k \sim \pi / h$ or $k R \leq 1$. Such a case corresponds to the elastic vibrations of the medium and is described by wave equations of theory of elasticity. The consideration of the size dependence of thermodynamic properties of nanocrystals will be carried out for the simplest type of nanocrystals, a thin plate. In this case, the dispersion relations have a rather simple appearance. A consistent consideration of elastic vibrations and propagation of elastic waves in plates was originally performed by Lamb [49] in the framework of general theory of elasticity.

Let us consider a plate of thickness $h$, with free surfaces parallel to the $(x, y)$ plane and the origin taken at the center of the plate. The $z$ axis is normal to the free surfaces $z= \pm h / 2$. The system is assumed to be of macroscopic size in the $(x, y)$ plane, so that vibrations can propagate as plane waves in the $x$ and $y$ directions. Note that we do not consider vibrations of the plate as a whole. The components of the stress tensor vanish at the free surfaces,

$$
\begin{aligned}
\left.\sigma_{z x}\right|_{ \pm h / 2} & =0, \\
\left.\sigma_{z z}\right|_{ \pm h / 2} & =0, \\
\left.\sigma_{z y}\right|_{ \pm h / 2} & =0 .
\end{aligned}
$$

For such a system, the displacement vector $\mathbf{u}$ can be represented as superposition of waves of the horizontal polarization, with $u_{x}=u_{z}=0, u_{y}=u$, 
and waves of the vertical polarization with $u_{x} \neq 0, u_{z} \neq 0$, and $u_{y}=0$. It is important that these two types of waves do not mix at the boundaries, so that they can be considered separately (see, e.g. Refs. [49, 50]).

Waves of the horizontal polarization are described by ordinary wave equations for the displacement $u_{y}$,

$$
\frac{\partial^{2} u_{y}}{\partial x^{2}}+\frac{\partial^{2} u_{y}}{\partial z^{2}}+\kappa^{2} u_{y}=0
$$

where $\kappa=\omega / c_{t}, \omega$ is an eigenfrequency, $c_{t}$ is transverse sound velocity. A solution of (11) in a particular case of a plane wave propagating along the $x$ direction is given by

$$
u_{y}=C \cos (\beta z-\delta) e^{i \xi x}
$$

Parameters $\beta$ and $\delta$ are found from both the boundary conditions (10) and the symmetry reasons allowing two types of the solutions, a symmetrical one, $u_{y}(z=h / 2)=u_{y}(z=-h / 2)$, and an antisymmetrical one, $u_{y}(z=h / 2)=$ $-u_{y}(z=-h / 2)$. These solutions lead generally to a dispersion relation given by

$$
\omega_{t}=c_{t} k_{n}
$$

with

$$
k_{n}=\sqrt{\xi^{2}+\zeta^{2}+\left(\frac{n \pi}{h}\right)^{2}}, \quad n=1,2,3, \ldots
$$

Here $\xi$ and $\zeta$ are, respectively, projections of the wave vector on the $x$ and $y$ axes for plane waves propagating in the $x y$ plane. The projection of the wave vector on the $z$ axis takes on discrete values,

$$
k_{z}=\frac{n \pi}{h} \text {. }
$$

In the case of waves of vertical polarization, the displacement is expressed in terms of scalar $\varphi$ and vector $\boldsymbol{\Psi}$ potentials,

$$
\mathbf{u}=\operatorname{grad} \varphi+\operatorname{rot} \boldsymbol{\Psi}
$$

The potential $\Psi$ can be chosen so that $\Psi_{y}=\Psi$ and $\Psi_{x}=\Psi_{z}=0$. Then $\varphi$ and $\Psi$ satisfy the wave equations,

$$
\begin{aligned}
\Delta \varphi+k^{2} \varphi & =0, \\
\Delta \Psi+\kappa^{2} \Psi & =0,
\end{aligned}
$$


where $k=\omega / c_{l}, c_{l}$ is longitudinal sound velocity. The solutions of Eqs. (16)(17) are given by

$$
\begin{aligned}
& \varphi=C \cos \left(\alpha z-\delta_{l}\right) e^{i \xi x}, \\
& \Psi=D \cos \left(\beta z-\delta_{t}\right) e^{i \xi x},
\end{aligned}
$$

where $\alpha$ and $\beta$ are real values given by

$$
\begin{aligned}
& \alpha=\sqrt{k^{2}-\xi^{2}}, \\
& \beta=\sqrt{\kappa^{2}-\xi^{2}} .
\end{aligned}
$$

In terms of these notations, the boundary conditions (10) are rewritten as

$$
\begin{aligned}
& \frac{\partial \Psi}{\partial z}-\left.i p \varphi\right|_{z= \pm h / 2}=0, \\
& \frac{\partial \varphi}{\partial z}+\left.i p \Psi\right|_{z= \pm h / 2}=0,
\end{aligned}
$$

where $p=\left(\xi^{2}-\beta^{2}\right) /(2 \xi)$. Hence we get the general dispersion relation for the acoustic part of the vibrational spectrum of the nanocrystal,

$$
\tan \left(\frac{\alpha h}{2}-\delta_{l}^{0}\right) \tan \left(\frac{\beta h}{2}-\delta_{t}^{0}\right)=\frac{p^{2}}{\alpha \beta} .
$$

From the symmetry reasons, the projections of the displacement vectors $u_{x}$ and $u_{z}$ should be either symmetrical or antisymmetrical. At the symmetrical conditions

$$
\delta_{l}^{0}=m \pi, \quad \delta_{t}^{0}=(2 n+1) \pi / 2, \quad m, n=0,1,2, \ldots
$$

the dispersion relation (24) appears as

$$
\cot \frac{\alpha h}{2} \tan \frac{\beta h}{2}=-\frac{p^{2}}{\alpha \beta} .
$$

The antisymmetrical condition is specified by

$$
\delta_{l}^{0}=(2 n+1) \pi / 2, \quad \delta_{t}^{0}=m \pi, \quad m, n=0,1,2, \ldots .
$$

leading to

$$
\tan \frac{\alpha h}{2} \cot \frac{\beta h}{2}=-\frac{p^{2}}{\alpha \beta}
$$




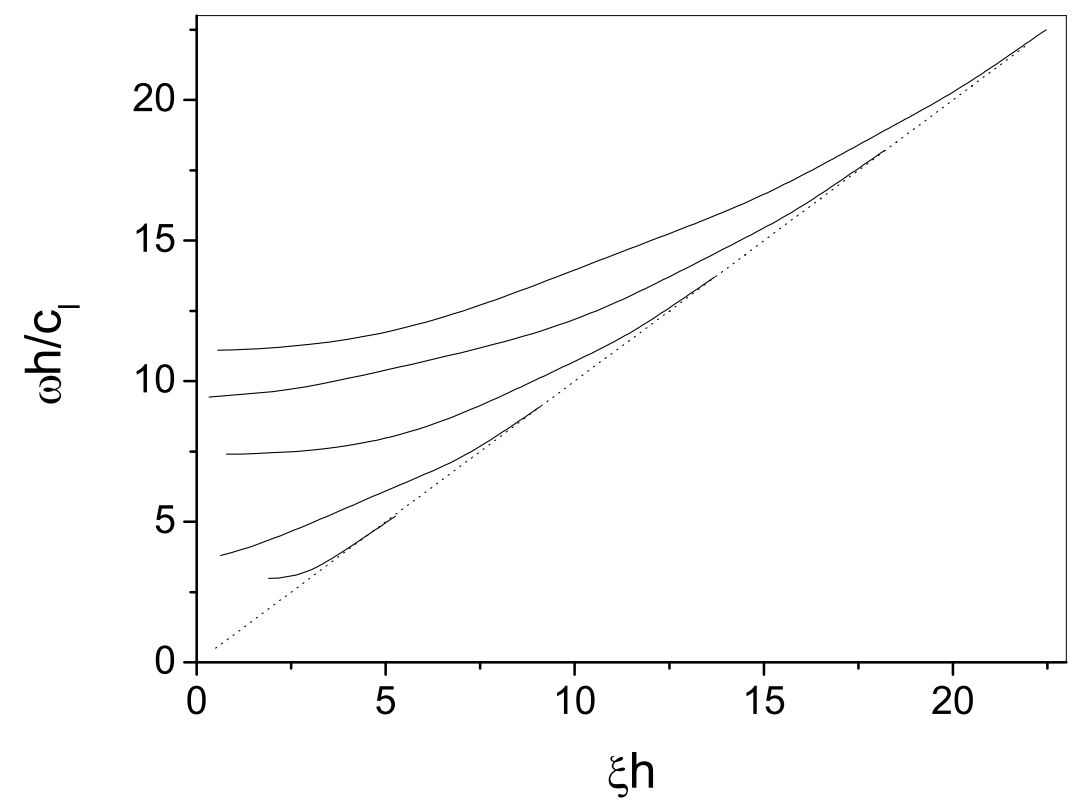

Figure 1: Dispersion curves of longitudinal vibrations of a thin plate for $n=1, \ldots 5$. The dash line corresponds to the dispersion relation $\omega=c_{l} \xi$ for the bulk crystal.

Note that satisfying the symmetrical condition for $u_{x}$ implies satisfying the antisymmetrical condition for $u_{z}$, and viceversa.

In Fig. 1 we plotted dispersion curves for the longitudinal characteristic vibrations of a thin plate obtained from (25). At a rather small thickness $h$ of the plate, the long-wave part $(k R \leq 1)$ of the vibrational spectrum of the nanocrystal differs substantially from that of the bulk crystal, described by the linear relation between the frequency and the wave vector, $\omega_{j}=c_{j} \xi$ ( $j=$ $l, t)$. The spectrum of the thin plate splits to separate vibration branches. For each branch, there is an integer number of wavelengths going across the plate thickness. The dispersion curves are well approximated by parabolas and can be analytically represented as

$$
\omega_{n}^{2}(\xi, \zeta)=\omega_{n, b}^{2}+\alpha_{n} c_{l}^{2}\left(\xi^{2}+\zeta^{2}\right) .
$$

Here $\omega_{n, b}$ is the minimal value of the frequency of the $n$th vibration branch, 
$\alpha_{n} \approx 1$. For each of both symmetrical (25) and antisymmetrical (26) cases we have two types of solutions for $\omega_{n, b}$ corresponding to longitudinal and transverse standing waves with the frequencies generally written as

$$
\omega_{n, b}^{j} \approx \frac{\pi n}{h} c_{j}, \quad j=l, t .
$$

As in the case of waves of the horizontal polarization (14), the wave vector for the both longitudinal and transverse waves of the vertical polarizations is given by Eq. (14), too.

A similarity of the expression for $\left(\omega_{n, b}^{j}\right)^{2}$ to the formula for the energy of a quantum particle in a one-dimensional potential well [51] is explained by the wave nature of both quantum particles and vibrational modes, exhibiting discreteness of the eigenvalues in the case of a small size of the system.

For the bulk fcc crystals, the first Brillouin zone has the form of a truncated octahedron and may be well approximated by a sphere of radius $k_{s}^{0}=\left(6 \pi^{2} / v\right)^{1 / 3}$. The radius $k_{s}^{0}$ is determined by the requirement that the number of independent values of the wave vector falling within the sphere is equal to the number of atoms of the simple lattice.

In the case of nanocrystals, evaluation of the radius $k_{s}$ of the spherical Brillouin zone requires taking into account the discrete character of $k_{z}$ in replacing summation over $\mathbf{k}$ by integration. This can be done using the Euler-Maclaurin formula [52]

$$
\sum_{k_{\min }}^{k_{\max }} f(k)=\int_{k_{\min }}^{k_{\max }} f(t) d t+\frac{1}{2}\left[f\left(k_{\min }\right)+f\left(k_{\max }\right)\right]+\ldots
$$

Then we get

$$
k_{s}=k_{s}^{0}\left(1+\frac{\pi}{4\left(k_{s}^{0} R\right)} \frac{R}{h}\right) .
$$

Presence of a size-dependent factor in (30) results in appearance of similar corrections to the coefficients $n_{l}$ in (3),

$$
n_{l}(h)=n_{l}^{0}\left(1+\gamma_{l} \frac{R}{h}\right)
$$

where $n_{l}^{0}$ is the bulk value of $n_{l}$, the first two coefficients $\gamma_{l}$ are $\gamma_{0}=0.68$ and $\gamma_{1}=0.74$. As will be shown below, the size dependence of the coefficients $n_{l}$ leads to a number of physical effects that should be observed in nanocrystals. 
It should be noted that the above procedure of calculation of the coefficients $\gamma_{l}$ seems to give overestimated values, because Eq. (30) for the quasidiscrete wave vector is valid, strictly speaking, only in the range $k R \leq 1$, sensible to the particle size. Near the boundaries of the Brillouin zone, the values of $\mathbf{k}$ are determined by the structure of the crystal lattice and are slightly dependent of the crystal size. However, the structure of Eq. (30) should remain, on the whole, the same, so that $\gamma_{l}$ may be considered as parameters of the theory.

\section{Thermodynamic properties of nanocrystals}

The equilibrium values of the quasi-elastic bond parameter $c_{0}$ and reduced lattice expansion $b_{0}$ are determined from minimization of the Gibbs function (8) with respect to $c$ and $b$,

$$
\left.\frac{\partial g}{\partial c}\right|_{\tau, p, b}=0,\left.\quad \frac{\partial g}{\partial b}\right|_{\tau, p, c}=0 .
$$

The condition for the equilibrium values of the variational parameters to exist is that

$$
D=\operatorname{det}\left|\begin{array}{cc}
g_{c c}^{\prime \prime} & g_{c b}^{\prime \prime} \\
g_{b c}^{\prime \prime} & g_{b b}^{\prime \prime}
\end{array}\right|>0
$$

The sufficient condition $D=0$ defines the point of anharmonic instability of the crystal where the minimum of $g(\tau, p, c, b)$ with respect to $c$ and $b$ disappears.

In the case of nanocrystals, the Gibbs free energy depends, besides temperature $\tau$ and pressure $p$, on the crystal size $h$. As a result, a number of thermodynamic characteristics of the crystal display size dependence. For example, the Debye temperature is directly proportional to the equilibrium value of the parameter $c_{0}(\tau, p, h)$ and is written as [44, 45]

$$
\Theta_{D} \approx\left(6 \sqrt{2} \pi^{2}\right)^{1 / 3}\left\langle\kappa_{j \mathbf{k}}\right\rangle A \Lambda c_{0}(\tau, p, h) / k_{B},
$$

where coefficients $\kappa_{j \mathbf{k}}=\omega_{j \mathbf{k}} /(k R)$ account for different polarizations of the acoustic waves, and $\left\langle\kappa_{j \mathbf{k}}\right\rangle \approx 0.67$ for the fcc crystals. The volume and temperature dependence of $\Theta_{D}$ determines the Grüneisen parameter,

$$
\gamma_{G}=-\frac{\left(\partial \ln \Theta_{D} / \partial \ln V\right)_{T}}{1-\left(\partial \ln \Theta_{D} / \partial \ln T\right)_{V}}
$$




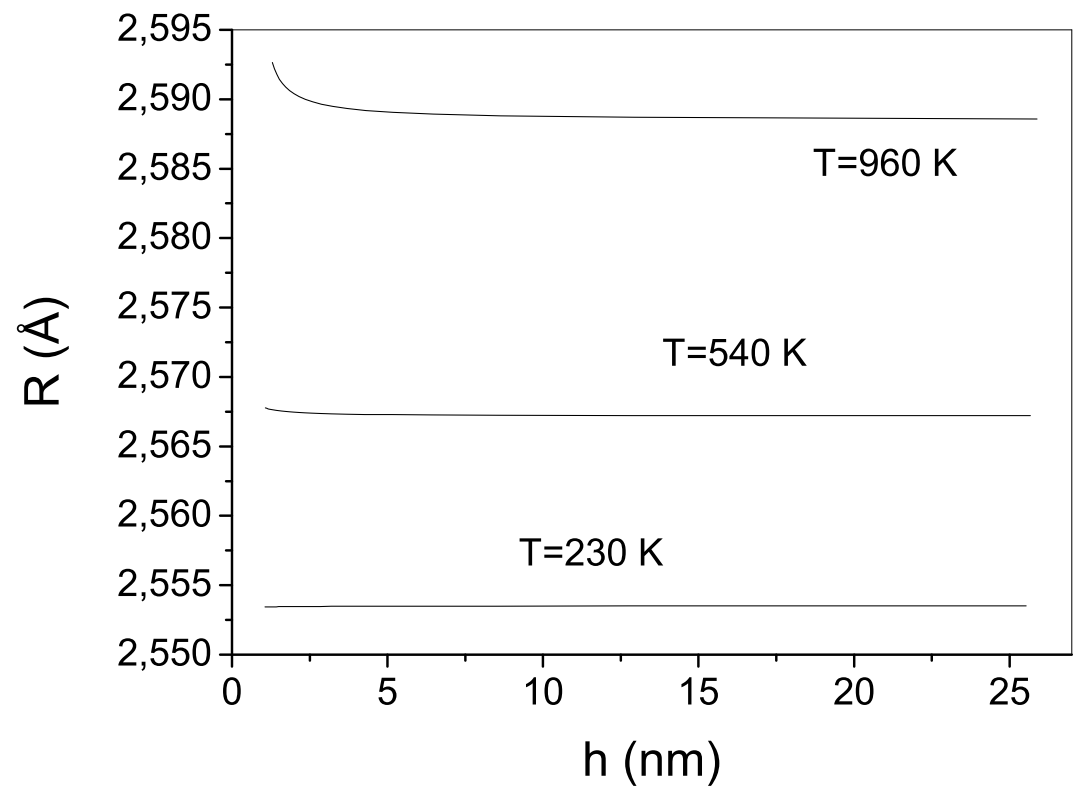

Figure 2: Size dependence of the interatomic distance in $\mathrm{Cu}$ nanocrystals at different temperatures.

The equilibrium value of $b_{0}(\tau, p, h)$ governs the interatomic distance, the coefficient of thermal expansion, and the bulk modulus of the nanocrystal. All these properties would depend on the crystal size.

If the melting temperature $T_{m}$ is assumed to be identical to the temperature of anharmonic instability of the crystal [39, 40], then it can be shown in the framework of the present statistical model that $T_{m}$ is related to the factor $n(\tau)$ as

$$
\tau_{m}=\frac{k_{B} T_{m}}{A} \sim \frac{1}{a_{3} n^{3}(\tau)} .
$$

Hence it follows that even a slight increasing of $n(\tau)$ accompanying reduction of the crystal size results in appreciable depression of the melting temperature of the nanocrystal. Since $R / h \ll 1$, it follows from (36) that

$$
\frac{T_{m}}{T_{m}^{b}}=1-\frac{\beta}{3 h}
$$


where $T_{m}^{b}$ is the melting temperature of the bulk material, and $\beta=9 \gamma_{0} R$. This is the well-known relationship between the relative melting temperature of a plate nanocrystal and its inverse size (see, e.g., [28]). The calculated value $\beta=1.59 \mathrm{~nm}$ for $\mathrm{Cu}$ is somewhat higher than that extracted from experimental data $[28](\beta \approx 0.9-1.2 \mathrm{~nm})$.

In Fig. 2 we plotted the interatomic distance in $\mathrm{Cu}$ nanocrystals versus particle size calculated from (32) at three values of temperature. Hereafter all theoretical results are presented for zero external pressure. A nanocrystal of size $h \approx 1.3 \mathrm{~nm}$ melts at $T=960 \mathrm{~K}$, which explains some nonlinear rise of $R(h)$ in the top curve. These results comply with the assumption [54, 55] that the size-induced lattice contraction observed in spherical nanoparticles is due to capillary pressure only.

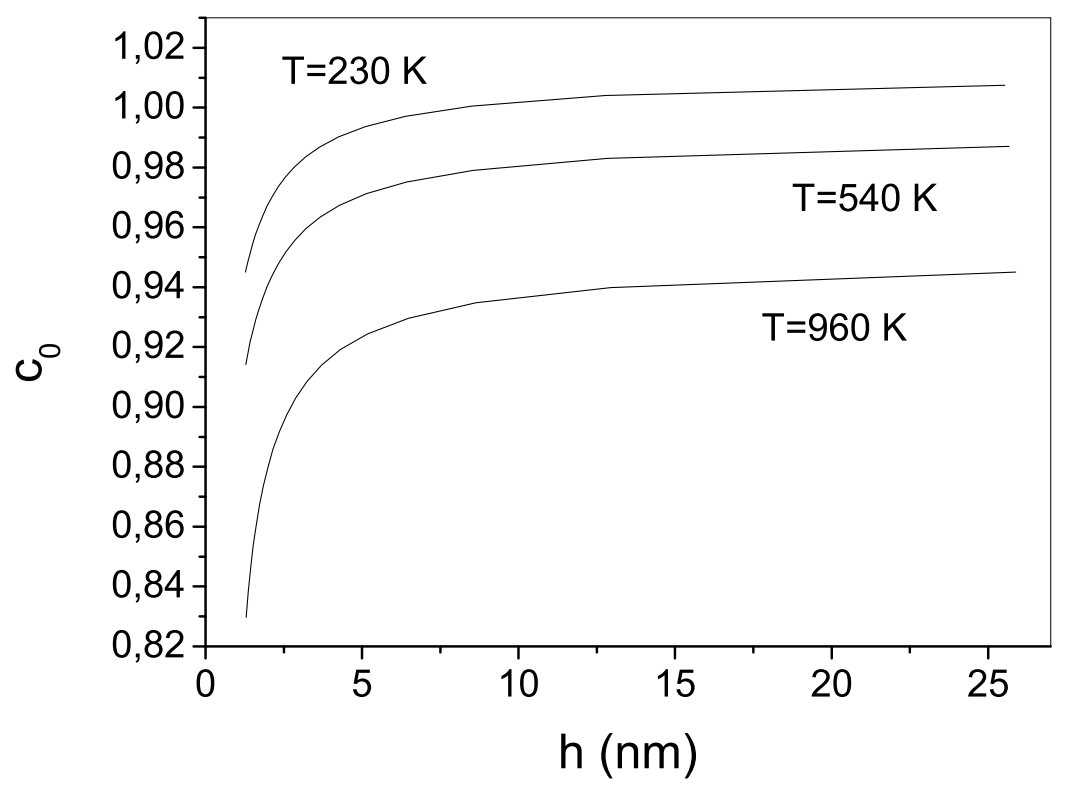

Figure 3: Size dependence of the effective quasi-elastic bond parameter $c_{0}(\tau, h)$ of $\mathrm{Cu}$ nanocrystals at different temperatures.

While the interatomic distances in the considered nanocrystals show no marked size dependence, the effective parameter $c_{0}(\tau, p, h)$ of the quasielastic bond varies substantially with $h$. Such dependence calculated for $\mathrm{Cu}$ 
nanocrystals is illustrated with Fig. 3 which demonstrates that $c_{0}$ decreases nonlinearly as $h$ decreases even at $T \ll T_{m}(h)$.

Size dependence of the Debye temperature $\Theta_{D}$ of small crystals was observed in a number of experimental studies [17, 19, 20]. In the framework of the present approach, $\Theta_{D}$ is proportional to the quasi-elastic bond parameter $c_{0}$ (34). The $\Theta_{D}(h)$ dependence presented in Fig. 4 agrees qualitatively with that obtained experimentally, e. g. for gold nanoparticles [19].

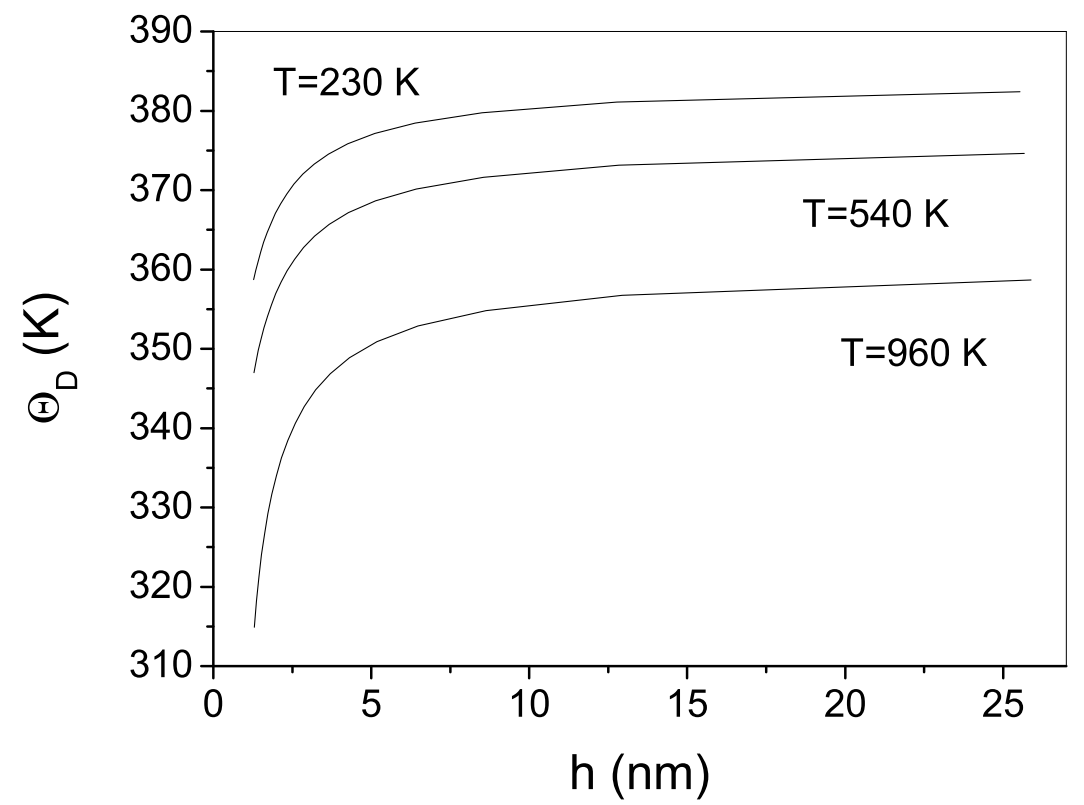

Figure 4: Size dependence of the Debye temperature of $\mathrm{Cu}$ nanocrystals at different temperatures.

In many bulk solids, the melting transition is preceded by anomalous behavior of temperature derivatives of thermodynamic functions (isobaric heat capacity, thermal expansion coefficient, Grüneisen parameter) due to evolution of the anharmonic instability in the phonon subsystem of the crystal as $T \rightarrow T_{m}$ [39, 40] (premelting phenomena). As seen from Fig. 5 for the isobaric heat capacity $C_{P}$, similar behavior should be also observed when the melting point is approached via reduction of the crystal size at constant temperature (the top curve). 


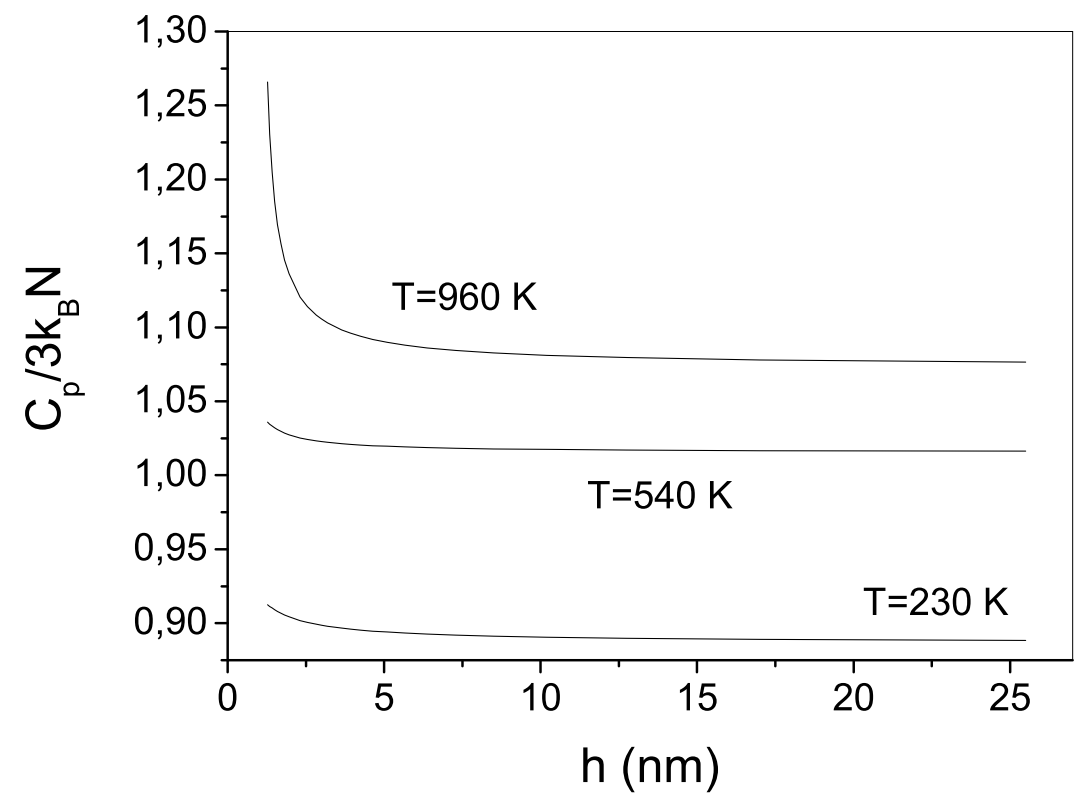

Figure 5: Size dependence of the isobaric heat capacity of $\mathrm{Cu}$ nanocrystals at different temperatures.

In Fig. 6] we show the melting temperature of a free $\mathrm{Cu}$ thin plate as a function of its thickness calculated from Eqs. (32) and the condition $D=0$, compared with that obtained from Eq. (36).

At present, the only generally recognized melting criterion is the empirical Lindemann rule suggesting that a solid melts when the rms displacement of atoms reaches a characteristic fraction of the interatomic distance. In terms of notations accepted in this work, the Lindemann ratio $\delta$ is given by

$$
\delta^{2}=\frac{\left\langle q^{2}\right\rangle}{R^{2}}=\frac{3 \tau}{4 c_{0}^{2}(\tau)(\alpha R)^{2} g_{t}} .
$$

Here $g_{t} \approx 0.77$ is the correlation smearing of the distribution width of an atom at a lattice site [43].

The size dependence of the Lindemann ratio is presented in Fig. 7 . A dramatic increase of the rms displacement of atoms in the premelting region is caused by nonlinear reduction of the quasi-elastic bond parameter $c_{0}(\tau, p, h)$ 


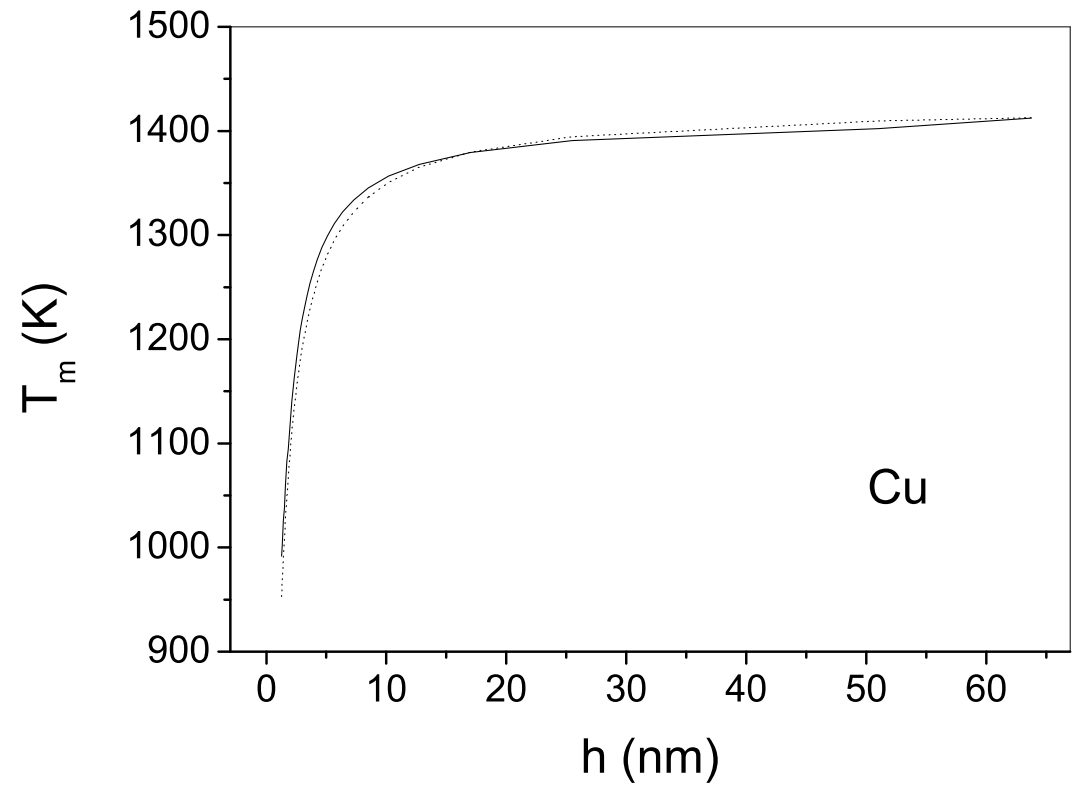

Figure 6: Melting temperature of a free $\mathrm{Cu}$ thin plate versus its thickness calculated by minimization of the Gibbs free energy (8) (solid curve) and from Eq. (36) (dotted curve).

in the vicinity of the instability point (Fig. 3). The maximal value $\delta \sim 0.1$ depends only slightly on $h$. Thus, as for the bulk solids, the premelting region is characterized by a nonlinear rise of some solid-state parameters of nanocrystals (isobaric heat capacity, the coefficient of thermal expansion, rms displacement etc.). It was also shown for the bulk case that the formation energy of the structural lattice defects exhibit a sharp drop just before $T_{m}$. All these premelting effects promote transition to a structurally disordered phase, i.e. melting.

Fig. 8 10 illustrate nonlinear behavior of thermodynamic properties of nanocrystals when the instability point is approached via increasing temperature at constant size. We see that the premelting range shifts as the crystal size decreases.

If two nanocrystals of the same thickness $h$ are brought into tight mechanical contact, the vibrational modes with wavelengths larger than the 


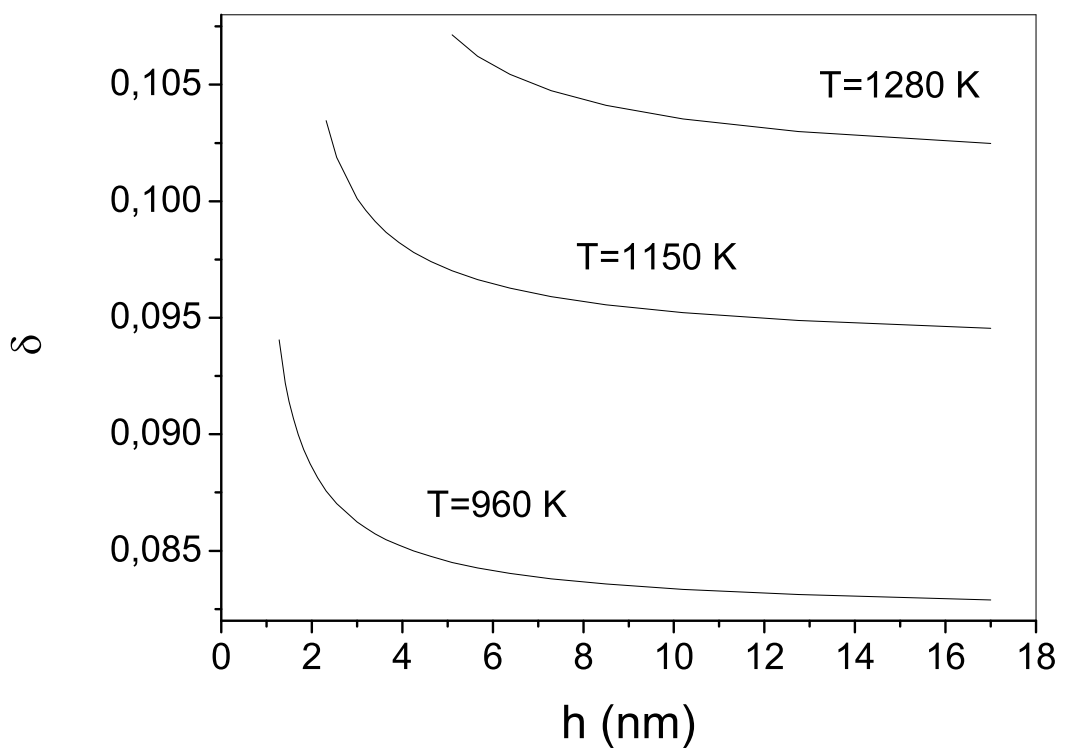

Figure 7: The relative rms displacement of atoms (the Lindemann ratio) of $\mathrm{Cu}$ thin plate versus its thickness at different temperatures.

characteristic thickness of the contact become collectivized, and their spectrum becomes, roughly speaking, equal to the spectrum of a nanocrystal with thickness $2 h$ [53]. Breaking the contact, i. e. separating the two plates, results in the reverse rearrangement of their vibrational spectra and is accompanied by a change $\Delta \varepsilon_{h}$ in the energy per atom $\varepsilon=g-\tau \partial g / \partial \tau$. Obviously, $\Delta \varepsilon_{h}=-\Delta \varepsilon_{2 h}$. If the nanoparticles are isolated, the energy change would lead to a change in the temperature of the nanocrystals, $\Delta T=\Delta \varepsilon_{h} / c_{V}$, where $c_{V}$ is isochoric heat capacity per atom of the nanocrystal. In Fig. 11 we plot a contact temperature change $\Delta T$ of two identical $\mathrm{Cu}$ thin plates versus initial temperature calculated for two values of the plate thickness, $h=5.1 \mathrm{~nm}$ and $h=1.26 \mathrm{~nm}$. As follows from the calculations, the temperature change is maximal if the initial system of separated nanoparticles is close to the melting point. 


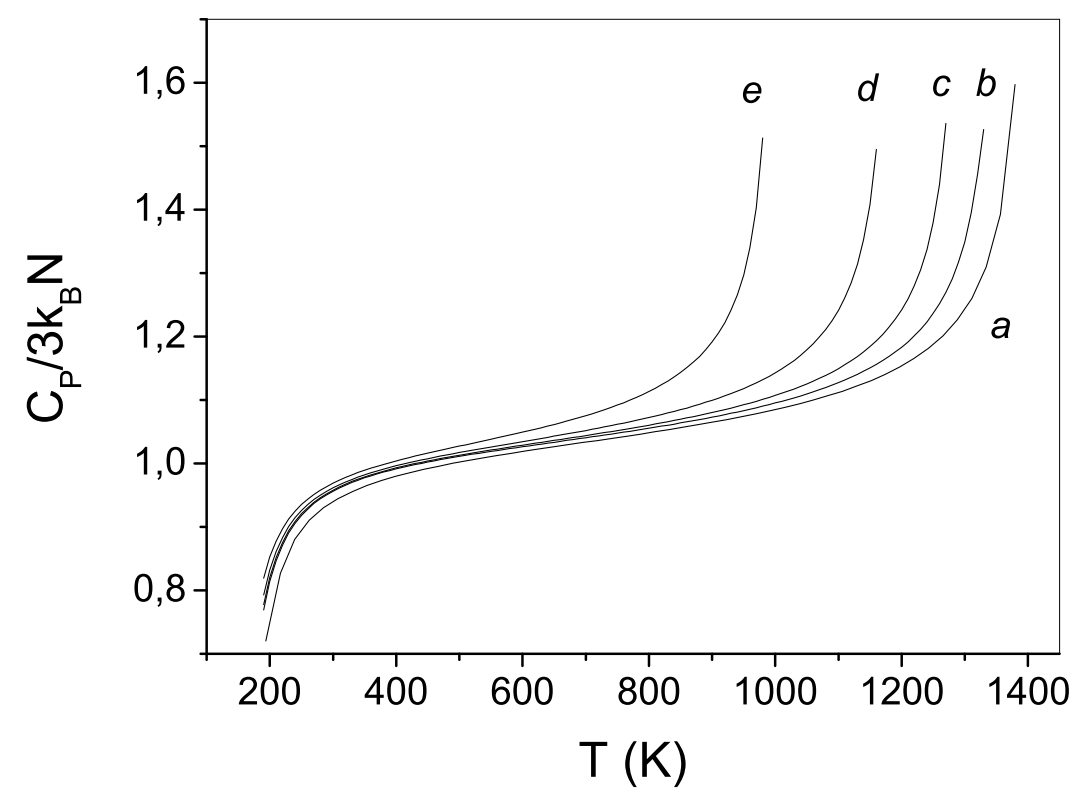

Figure 8: The temperature dependence of the isobaric heat capacity of $\mathrm{Cu}$ nanocrystals: (a) bulk crystal; (b) $h=10.2 \mathrm{~nm}$; (c) $h=5.1 \mathrm{~nm}$; (d) $h=$ $2.55 \mathrm{~nm}$; (e) $h=1.26 \mathrm{~nm}$.

\section{Discussion}

So far there is a large amount of experimental data concerning size influence on the bulk properties of nanoparticles, such as cohesion energy [17, 18], Debye temperature [17, 19, 20], and activation energy of diffusion [21, 22]. One of the most pronounced manifestations of change of thermodynamic properties observed in nanosized crystalline systems is the effect of reduction of the melting temperature of free nanocrystals [2]-[16]. So the principal problem of statistical description of thermodynamics of nanocrystals is elucidation of the mechanism of size effect on statistical characteristics of atoms in such systems. Relying on the results of molecular dynamics simulations of thermodynamic properties and melting of nanocrystals [35, 36], it is natural to conclude that a thin (relative to the particle size) surface layer has only a minor influence upon the bulk properties of the particles; moreover, in the 


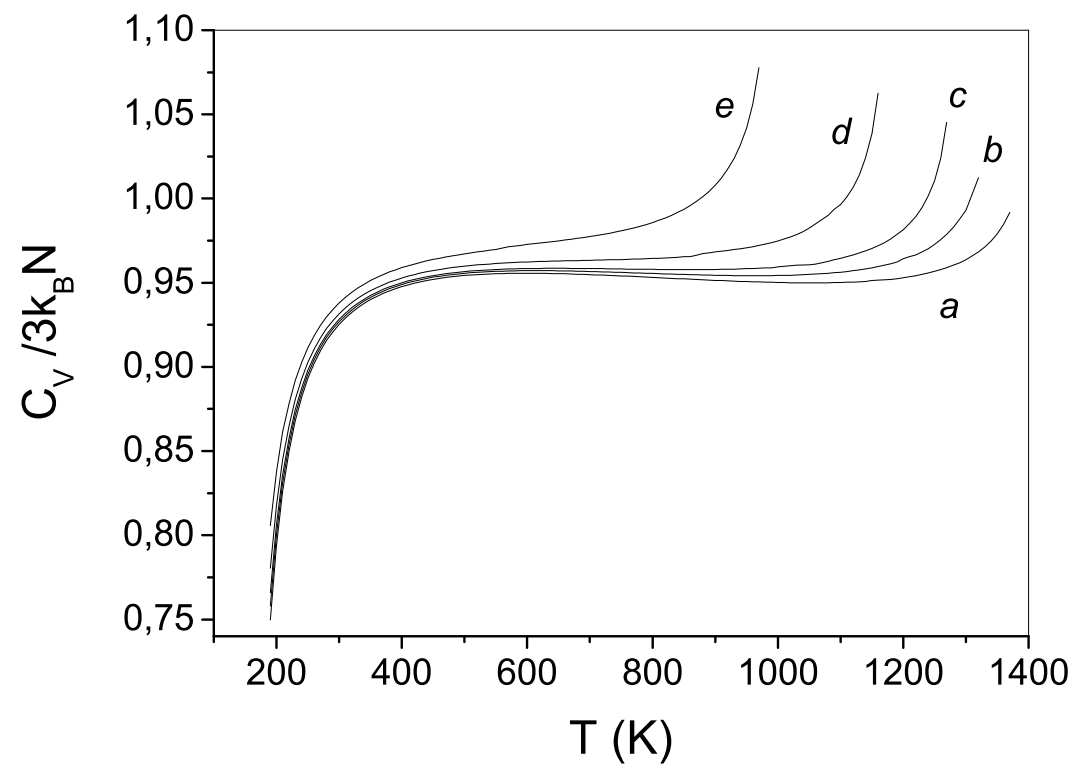

Figure 9: The temperature dependence of the isochoric heat capacity of $\mathrm{Cu}$ nanocrystals: (a) bulk crystal; (b) $h=10.2 \mathrm{~nm}$; (c) $h=5.1 \mathrm{~nm}$; (d) $h=2.55 \mathrm{~nm} ;(\mathrm{e}) h=1.26 \mathrm{~nm}$.

case of spherical particles an additional capillary pressure should contribute to increasing of both the melting temperature and the Debye temperature.

It is shown in this work that an important size-dependent factor governing thermodynamic behavior of a nanocrystal is discreteness of its vibrational spectrum. It leads to increasing of the parameter $n(\tau)$ of the statistical distribution function of atomic coordinates (21) as crystal's size decreases. As a consequence, the average value of the interaction energy of atoms in the nanocrystal changes with its size. It is necessary to emphasize that such influence on a crystalline system mediated by direct change of the statistical distribution function is inherent only in nanosystems. In this connection, it is worth noting a specific character of thermodynamic response of a nanocrystal to variation of its size. As the crystal's size decreases, the parameter $c_{0}(\tau, p, h)$ of quasi-elastic bond of its atoms decreases (Fig. 3), as well as the Debye temperature (Fig. 4), while interatomic distance remains nearly con- 


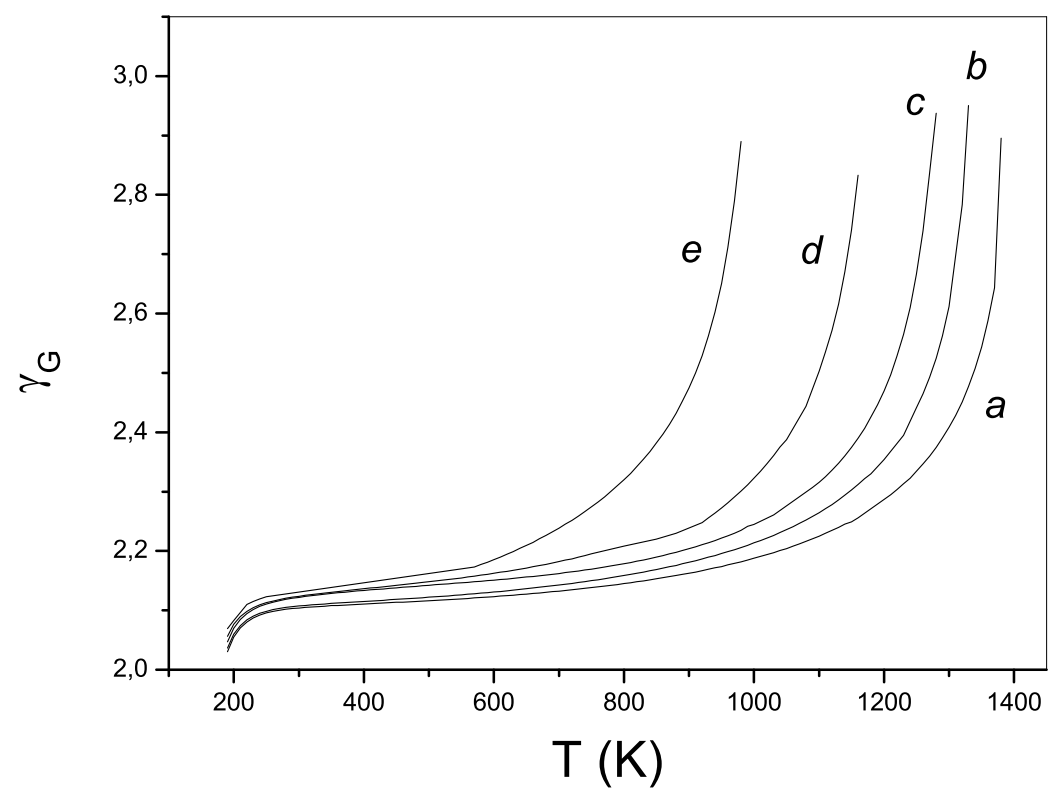

Figure 10: The temperature dependence of the Grüneisen parameter of $\mathrm{Cu}$ nanocrystals: (a) bulk crystal; (b) $h=10.2 \mathrm{~nm}$; (c) $h=5.1 \mathrm{~nm}$; (d) $h=$ $2.55 \mathrm{~nm} ;(\mathrm{e}) h=1.26 \mathrm{~nm}$.

stant (Figs. 22). The melting temperature (36) of the nanocrystal is markedly decreased (Figs. 6). Independently of the crystal size, the melting transition occurs when the Lindemann criterion is satisfied (Fig. 7). Along with a sizedependent shift of the melting temperature, there is also a corresponding shift of the premelting range where thermodynamic properties (isobaric heat capacity, coefficient of thermal expansion etc.) display nonlinear behavior.

\section{Acknowledgement}

This work was supported in part by Award no. 28/08-H in the framework of the Complex Program of Fundamental Investigations "Nanosized systems, nanomaterials, nanotechnology" of National Academy of Sciences of Ukraine. 


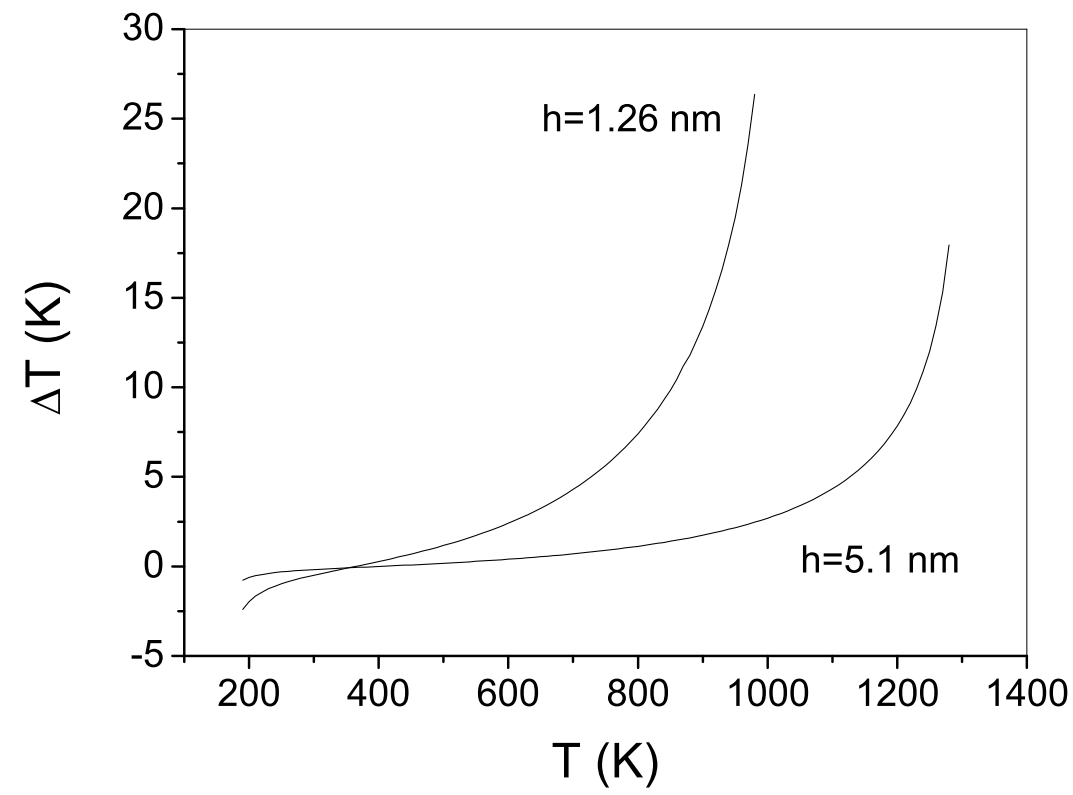

Figure 11: A temperature change at mechanical contact of two identical $\mathrm{Cu}$ thin plates of thickness $h$ versus initial temperature.

\section{References}

[1] P. Pawlow, Z. Phys. Chem. 65, 1 (1909); 65, 545 (1909).

[2] M. Takagi, J. Phys. Soc. Japan 9, 359 (1954).

[3] C. R. M. Wronski, Br. J. Appl. Phys. 18, 1731 (1967).

[4] C. J. Coombes, J. Phys. F: Met. Phys. 2, 441 (1972).

[5] N. T. Gladkich, R. Neidermayer, and K. Spiegel, Phys. Stat. Sol., 15, 181 (1966).

[6] Ph. Buffat and J.-P. Borel, Phys. Rev. A 13, 2287 (1976).

[7] R. H. Willens, A. Kornblit, L. R. Testardi, and S. Nakahara, Phys. Rev. B 25, 290 (1982). 
[8] S. L. Lai, J. Y. Guo, V. Petrova, G. Ramanath, and L. H. Allen, Phys. Rev. Lett. 77, 99 (1996).

[9] S. L. Lai, J. R. A. Carlsson, and L.H. Allen, Appl. Phys. Lett. 72, 1098 (1998).

[10] M. Zhang, M. Yu. Efremov, F. Schiettekatte, E. A. Olson, A. T. Kwan, S. L. Lai, T. Wisleder, J. E. Greene, and L. H. Allen, Phys. Rev. B 62, 10548 (2000).

[11] E. A. Olson, M. Yu. Efremov, M. Zhang, and L. H. Allen, J. Appl. Phys. 97, 034304 (2005).

[12] M. Yu. Efremov, F. Schiettekatte, M. Zhang, E. A. Olson, A. T. Kwan, R. S. Berry, and L. H. Allen, Phys. Rev. Lett. 85, 3560 (2000).

[13] M. Zhang, M. Yu. Efremov, E. A. Olson, Z. S. Zhang, and L. H. Allen, Appl. Phys. Lett. 81, 3801 (2002).

[14] J. Zhong, L. H. Zhang, Z. H. Jin, M. L. Sui and K. Lu, Acta Mater. 49, 2897 (2001).

[15] T. Bachels, H.-J. Guntherodt, and R. Schafer, Phys. Rev. Lett. 85, 1250 (2000).

[16] G. A. Breaux, R. C. Benirschke, and M. F. Jarrold, J. Chem. Phys. 121, $6502(2004)$.

[17] C. C. Yang and S. Li, Phys. Rev. B 75, 165413 (2007).

[18] W. H. Qi and M. P. Wang, J. Mater. Sci. Lett. 21, 1743 (2002).

[19] G. Kästle, H.-G. Boyen, A. Schröder, A. Plettl, and P. Ziemann, Phys. Rev. B 70, 165414 (2004).

[20] C. C. Yang, M. X. Xiao, W. Li, and Q. Jiang, Solid State Commun. 139, 148 (2006).

[21] T. Shibita, B. A. Bunker, Z. Zhang, D. Meisel, C. F. Vardeman II, and J. D. Gezelter, J. Am. Chem. Soc. 124, 11989 (2002).

[22] Q. Jiang, S. H. Zhang, and J. C. Li, J. Phys. D 37, 102 (2004). 
[23] T. Korhonen, M. J. Puska, and R. M. Nieminen, Phys. Rev. B 51, 9526 (1995).

[24] C. J. Zhang and A. Alavi, J. Am. Chem. Soc. 127, 9808 (2005)

[25] K.-J. Hanszen, Z. Phys. 157, 523 (1960).

[26] P. R. Couchman and W. A. Jesser, Nature (London) 269, 481 (1977).

[27] K. F. Peters, J. B. Cohen, and Y.-W. Chung, Phys. Rev. B 57, 13430 (1998).

[28] K. K. Nanda, S. N. Sahu, and S. N. Behera, Phys. Rev. A 66, 013208 (2002).

[29] J. Tateno, Solid State Commun. 10, 61 (1972).

[30] Y. Wang, S. Teitel, and Christoph Dellago, Chem. Phys. Lett. 394, 257 (2004).

[31] Y. Qi, T. Cagin, W. L. Johnson, and W. A. Goddard III, J. Chem. Phys. 115, 385 (2001).

[32] L. Hui, F. Pederiva, B. L. Wang, J. L. Wang, and G. H. Wang, Appl. Phys. Lett. 86, 011913 (2005).

[33] U. Tartaglino, T. Zykova-Timan, F. Ercolessi, and E. Tosatti, arXiv:cond-mat/0504680 v2: 3 Aug 2005.

[34] F. Ercolessi, W. Andreoni, and E. Tosatti, Phys. Rev. Lett. 66, 911 (1991).

[35] F. Delogu, Phys. Rev. B 72, 205418 (2005).

[36] F. Delogu, Phys. Rev. B 75, 235421 (2007).

[37] S. J. Zhao, S. Q. Wang, D. Y. Cheng, and H. Q. Ye, J. Phys. Chem. B 105, 12857 (2001).

[38] F. Baletto and R. Ferrando, Rev. Mod. Phys. 77, 371 (2005).

[39] A. I. Karasevskii and V. V. Lubashenko, Phys. Rev. B 71, 012107 (2005). 
[40] A. I. Karasevskii, W. B. Holzapfel, and V. V. Lubashenko, J. Low Temp. Phys. 139, 609 (2005).

[41] S. A. Gurvits, A. A. Migdal, and A. M. Polyakov, Zh. Exp. Teor. Fiz. 46, 213 (1964).

[42] M. A. Krivoglaz and A. I. Karasevskii, Fiz. Tverd. Tela 17, 2565 (1975) [Sov. Phys. Solid State, 17, 1709 (1976)].

[43] A. I. Karasevskii and V.V. Lubashenko, Phys. Rev. B 66, 054302 (2002).

[44] A. I. Karasevskii and W. B. Holzapfel, Phys. Rev. B 67, 224301 (2003).

[45] A. I. Karasevskii and W. B. Holzapfel, Low Temp. Phys. 29, 711 (2003).

[46] A. I. Karasevskii and V. V. Lubashenko, Phys. Stat. Sol. (b) 241, 1274 (2004).

[47] A. I. Karasevskii and V.V. Lubashenko, Low Temp. Phys. 33, 578 (2007).

[48] R. P. Feynmann, Statistical Mechanics (Benjamin, New York, 1972).

[49] H. Lamb, Proc. Math. Soc. London 98, 205 (1920).

[50] A.E.H. Love, Treatise on the Mathematical Theory of Elasticity (New York, Dover, 1944).

[51] L. D. Landau and E.M. Lifshitz, Quantum Mechanics (Pergamon Press, Oxford, 1965).

[52] E. Madelung, Mathematical Apparatus of Physics (Springer, Berlin, 1957).

[53] Physical acoustics. Principles and Methods, edited by W. P. Mason (Academic Press, New York and London, 1968), Vol. 4, Applications to Quantum and Solid State Physics. Part B.

[54] K. K. Nanda, S. N. Behera, and S. N. Sahu, J. Phys.: Condens. Matter 13, 2861 (2001).

[55] W. H. Qi and M. P. Wang, J. Nanopart. Res. 7, 51 (2005). 